\title{
A utilização da fonoforese na dor e funcionalidade em indivíduos com osteoartrite de joelho: Uma revisão de escopo
}

The use of phonophoresis in pain and functionality in individuals with knee osteoarthritis: A review of the scope

El uso de la fonoforesis en el dolor y la funcionalidad en personas con osteoartritis de rodilla: Una revisión del alcance

Universidade Federal do Amazonas, Brasil

E-mail: rodriguesjeancarlos2@gmail.com E-mail: rdelima32@gmail.com

Johrdy Amilton da Costa Braga ORCID: https://orcid.org/0000-0003-2020-250X

Universidade Federal do Amazonas, Brasil E-mail: johrdybraga@gmail.com

Alexandre Freitas dos Santos

ORCID: https://orcid.org/0000-0001-9670-5985

Universidade Federal do Amazonas, Brasil

E-mail: alexandreisfufam@gmail.com

Eduardo Teixeira de Melo

ORCID: https://orcid.org/0000-0002-1154-8421

Universidade Federal do Amazonas, Brasil E-mail: edemelo798@gmail.com

Kaio de Souza Trindade

ORCID: https://orcid.org/0000-0002-3250-6394

Universidade Federal do Amazonas, Brasil

E-mail: kaio.strindade@gmail.com

Romário de Lima Nascimento ORCID: https://orcid.org/0000-0003-1377-071X

Universidade Federal do Amazonas, Brasil E-mail: rdelima32@gmail.com

Vinicius Amaral Praia

ORCID: https://orcid.org/0000-0003-4561-1414

Universidade Federal do Amazonas, Brasil

E-mail: vkapraia@gmail.com

Thiago Santos da Silva

ORCID: https://orcid.org/0000-0001-8291-1470

Universidade Federal do Amazonas, Brasil

E-mail: thiagofisioufam@gmail.com

Adriano Carvalho de Oliveira

ORCID: https://orcid.org/0000-0001-8225-7974

Universidade Federal do Amazonas, Brasil

E-mail: acofisioufam@gmail.com

\begin{abstract}
Resumo
O objetivo desta revisão de escopo foi mapear e sintetizar evidências a respeito da utilização da fonoforese para a dor e funcionalidade na osteoartrite de joelho, assim como buscar quais os parâmetros/fármacos estão sendo utilizados nesta técnica. Os bancos de dados utilizadas para a busca dos artigos foram: PubMed, Biblioteca Virtual em Saúde, SciELO, PEDro, e fontes de literatura cinzenta, publicadas no período entre abril de 2011 a março de 2021. Foram considerados estudos realizados em indivíduos com osteoartrite, que foram tratados com fonoforese para a dor e funcionalidade, em qualquer contexto clínico e geográfico. Após as buscas, foram inclusos nesta revisão 7 artigos, os quais apontam resultados positivos da fonoforese nos impactos da osteoartrite de joelho. Em conclusão, a fonoforese é eficaz no alivio da dor e melhora da funcionalidade em indivíduos portadores de osteoartrite de joelho.
\end{abstract}

Palavras-chave: Dor; Fonoforese; Modalidades de fisioterapia; Osteoartrite do joelho. 


\begin{abstract}
The objective of this scope review was to map and synthesize evidence regarding the use of phonophoresis for pain and functionality in knee osteoarthritis, as well as to seek which parameters/drugs are being used in this technique. The databases used to search for articles were: PubMed, Virtual Health Library, SciELO, PEDro, and gray literature sources, published in the period between April 2011 and March 2021. Studies carried out in individuals with osteoarthritis were considered, who were treated with phonophoresis for pain and functionality, in any clinical and geographic context. After the searches, 7 articles were included in this review, which show positive results of phonophoresis in the impacts of knee osteoarthritis. In conclusion, phonophoresis is effective in relieving pain and improving functionality in individuals with knee osteoarthritis.
\end{abstract}

Keywords: Pain; Phonophoresis; Physiotherapy modalities; Knee osteoarthritis.

\title{
Resumen
}

El objetivo de esta revisión de alcance fue mapear y sintetizar la evidencia sobre el uso de la fonoforesis para el dolor y la funcionalidad en la osteoartritis de rodilla, así como buscar qué parámetros / fármacos se están utilizando en esta técnica. Las bases de datos utilizadas para la búsqueda de artículos fueron: PubMed, Virtual Health Library, SciELO, PEDro y fuentes de literatura gris, publicadas en el período comprendido entre abril de 2011 y marzo de 2021 . Se consideraron estudios realizados en individuos con artrosis, que fueron tratados con fonoforesis. para el dolor y la funcionalidad, en cualquier contexto clínico y geográfico. Tras las búsquedas, se incluyeron en esta revisión 7 artículos, que muestran resultados positivos de la fonoforesis en los impactos de la osteoartritis de rodilla. En conclusión, la fonoforesis es eficaz para aliviar el dolor y mejorar la funcionalidad en personas con osteoartritis de rodilla.

Palabras clave: Dolor; Fonoforesis; Modalidades de fisioterapia; Osteoartritis de rodilla.

\section{Introdução}

As ciências da saúde estão constantemente em busca de soluções e alternativas através de pesquisas científicas, estudos, averiguações e até mesmo na prática clínica que comprovem a eficiência de métodos menos invasivos, menos dolorosos que não gerem transtornos aos usuários dos serviços de saúde no tratar de doenças (Boyaci et al., 2013; Prentice, 2014; Pinkaew et al., 2020). Neste cenário, temos a osteoartrite, que é uma doença que possui a determinação de sua prevalência intimamente ligada ao sexo, articulação acometida, e principalmente a idade da população estudada (Ito et al., 2019; Silva et al., 2019). Sendo que no Brasil, de forma geral atinge aproximadamente $4,14 \%$ da população (Senna et al., 2004).

A osteoartrite é uma doença reumática degenerativa e progressiva, que atinge as articulações sinoviais, causando diversas alterações na cartilagem articular, além do estreitamento do espaço intra-articular (Barduzzi et al., 2013; Yamada et al., 2018). Essa doença pode evoluir para uma deformidade da articulação com a formação de osteófitos, os quais provocam dor, tumefação, rigidez articular na movimentação e refletem algum grau de sinovite e crepitação, indicativos de lesão cartilaginosa que evolui com o tempo (Barduzzi et al., 2013; Magalhães, 2018; Yamada et al., 2018).

Os primeiros sintomas são dor, rigidez matinal, diminuição ou perda de movimento, crepitações, edema, fraqueza e espasmos musculares (Barduzzi et al., 2013; Magalhães, 2018; Yamada et al., 2018), gerando alterações funcionais subjetivas, como diminuição da velocidade da marcha e dificuldades para subir e descer escadas (Duarte et al., 2013; Yamada et al., 2018).

A osteoartrite é uma resposta complexa dos tecidos articulares à idade, fatores genéticos e ambientais. O processo degenerativo é complexo e inicia- se com o envelhecimento, ou podem ocorrer fatos na vida do indivíduo que provoquem o início precoce desse processo degenerativo natural, como as doenças inflamatórias ou infecciosas que destroem a estrutura cartilaginosa, traumas ou cirurgias que envolvem a cartilagem, precipitando a osteoartrite (Barduzzi et al., 2013; Luksurapan et al., 2013; Zhao et al., 2016).

É comum a ocorrência da osteoartrite em articulações que sofrem maior descarga de peso corporal, como os joelhos, pois se existir movimento na articulação acometida o osso subcondral torna-se esclerótico, por outro lado, se não existe 
movimento considerável o osso e toda a musculatura adjacente sofre atrofia (Benlidayi et al., 2018; Magalhães, 2018). Como consequência, esse fator leva a uma maior progressão da doença, pois os músculos periarticulares tem papel fundamental na absorção de choques e na estabilização da articulação (Yamada et al., 2018; Ahmed et al., 2019).

A associação entre a osteoartrite de joelho e a obesidade também pode gerar maiores níveis de dor e de dificuldades funcionais, especialmente nas atividades de locomoção, que exigem movimentação e descarga de peso sobre as articulações afetadas (Vasconcelos et al., 2006; Boonhong et al., 2018).

O principal objetivo do tratamento da osteoartrite é obter o controle da dor e rigidez nas articulações para melhorar a funcionalidade. A fisioterapia vem se destacando nos processos de reabilitação com uso de técnicas não invasivas que diminuem os transtornos aos pacientes (Prentice, 2014; Wu et al., 2019; Ahmed et al., 2019; Pinkaew et al., 2020).

Estudos demonstraram que o ultrassom terapêutico promove a formação de colágeno, induz a reparação da cartilagem e regula as respostas inflamatórias por meio da estimulação de efeitos térmicos que aumentam o fluxo sanguíneo na região de tratamento. Isso leva à redução da dor e de espasmos musculares, além do aumento da extensibilidade tecidual e melhora do fluxo sanguíneo local (Prentice, 2014; Wu et al., 2019).

O ultrassom terapêutico pode ser utilizado para a facilitação do transporte de substâncias em tecidos humanos, além da absorção das mesmas. Dessa forma, surgiram algumas técnicas utilizadas em reabilitação com esse aparelho, dentre elas destaca-se a fonoforese, que é o emprego de anti-inflamatórios tópicos em forma de gel/creme e ultrassom terapêutico, usados simultaneamente (Cavalcante et al., 2019). A fonoforese é uma técnica que facilita a penetração cutânea através da energia ultrassônica na desorganização da estrutura lipídica da epiderme, utilizando como meio de contato entre o cabeçote do aparelho e a pele, gel ou medicamento (Prentice, 2014; Cavalcante et al., 2019). Esse método evita efeitos colaterais sistêmicos, uma vez que a droga empregada age no local da área a ser tratada. Embora a administração de drogas através da pele tenha se mostrado uma alternativa importante, sua utilização limita-se pela escassez de drogas com propriedades satisfatórias. Ademais, preparações tópicas que possuem baixo índice de transmissibilidade podem diminuir a efetividade da terapia com ultrassom (Cavalcante et al., 2019).

Há evidências que sustentam a aplicação da fonoforese na osteoartrite de joelho (Boyaci et al., 2013; Luksurapan et al., 2013; Zhao et al., 2016; Benlidayi et al., 2018; Boonhong et al., 2018; Ahmed et al., 2019; Pinkaew et al., 2020), embora haja divergências na literatura (Cavalcante et al., 2019). Assim, o objetivo da presente revisão foi mapear e sintetizar evidências a respeito da utilização da fonoforese para a dor e funcionalidade na osteoartrite de joelho, assim como buscar quais os parâmetros/fármacos estão sendo utilizados nesta técnica.

\section{Metodologia}

O presente estudo é uma revisão de escopo da literatura. Apesar de não seguir estritamente, utilizou-se a metodologia do JBI Collaboration (JBI) para guiar a presente revisão (Peters et al., 2020).

\subsection{Pergunta de revisão}

As perguntas que nortearam esta revisão foram:

Quais as evidências disponíveis a respeito da fonoforese na dor e na funcionalidade em indivíduos portadores de osteoartrite de joelho?

Quais parâmetros/fármacos estão sendo usados na fonoforese? 


\subsection{Critérios de elegibilidade}

Os critérios de elegibilidade foram elaborados baseados no mnemônico PCC (participantes, conceito e contexto), seguindo a recomendação do JBI.

\subsubsection{Participantes}

Considerou-se estudos realizados em pessoas com osteoartrite de joelho, independente da sua etiologia, de sexo e de raça, com idade superior ou igual a 18 anos.

\subsubsection{Conceitos}

A revisão considerou estudos que trouxessem a utilização da fonoforese na dor e na funcionalidade em portadores de osteoartrite de joelho (Boyaci et al., 2013; Luksurapan et al., 2013; Zhao et al., 2016; Boonhong et al., 2018; Ahmed et al., 2019; Pinkaew et al., 2020).

\subsubsection{Contextos}

Considerou-se estudos produzidos em quaisquer cenários de atenção à saúde, como no ambulatorial, hospitalar e até mesmo na atenção básica, em qualquer contexto geográfico e socioeconômico.

\subsubsection{Tipos de fontes}

Esta revisão considerou somente estudos quantitativos experimentais, como ensaios clínicos randomizados, ensaios clínicos controlados, ensaios pragmáticos e estudos quase-experimentais ou do tipo antes e depois, publicados nos idiomas inglês, português e espanhol (uma vez que são idiomas abarcados pelos autores), entre entre abril de 2011 e março de 2021.

\subsection{Estratégias de busca}

Foram elaboradas estratégias de busca adaptadas para cada fonte de informação, e essas estratégias e seus respectivos resultados estão registrados no Quadro 1. Foram empregados descritores controlados de acordo com DeCS/MeSH (Descritores em Ciências da Saúde / Medical Subject Headings) e descritores não controlados. Em casos de indisponibilidade do texto completo os autores do estudo foram contatados.

Quadro 1. Estratégia de busca nas fontes de informação.

\begin{tabular}{|c|c|l|}
\hline Fontes de informação & Itens encontrados & \multicolumn{1}{c|}{ Busca (Consulta) } \\
\hline PubMed & 183.734 & (((phonophoresis) AND (osteoarthritis)) AND (knee Joint)) OR (knee) \\
\hline $\begin{array}{c}\text { Biblioteca Virtual de } \\
\text { Saúde (BVS) }\end{array}$ & 16 & $\begin{array}{l}\text { (fonoforese) OR (phonophoresis) OR (fonoforesis) AND (osteoartrite) OR } \\
\text { (osteoarthritis) OR (osteoartritis) AND (articulação do joelho) OR (knee Joint) OR } \\
\text { (articulación de la rodilla) OR (joelho) OR (knee) OR (rodilla) }\end{array}$ \\
\hline $\begin{array}{c}\text { Scientific Electronic } \\
\text { Library Online (SciELO) }\end{array}$ & 0 & $\begin{array}{l}\text { (fonoforese) OR (phonophoresis) OR (fonoforesis) AND (osteoartrite) OR } \\
\text { (osteoarthritis) OR (osteoartritis) AND (articulação do joelho) OR (knee Joint) OR } \\
\text { (articulación de la rodilla) OR (joelho) OR (knee) OR (rodilla) }\end{array}$ \\
\hline $\begin{array}{c}\text { Physiotherapy Evidence } \\
\text { Database (PEDro) }\end{array}$ & 0 & phonophoresis* osteoarthritis* knee Joint* \\
\cline { 2 - 4 } $\begin{array}{c}\text { Catálogo de Teses e } \\
\text { Dissertações da CAPES } \\
\text { Brasil (CTD) }\end{array}$ & 0 & $\begin{array}{l}\text { fonoforese OR phonophoresis AND osteoartrite OR osteoarthritis AND } \\
\text { articulação do joelho OR knee Joint OR joelho OR knee }\end{array}$ \\
\hline $\begin{array}{c}\text { Sumários de Revistas } \\
\text { Brasileiras (Sumários.org) }\end{array}$ & 0 & $\begin{array}{l}\text { fonoforese OR phonophoresis AND osteoartrite OR osteoarthritis AND } \\
\text { articulação do joelho OR knee Joint OR joelho OR knee }\end{array}$ \\
\hline $\begin{array}{c}\text { Biblioteca Digital } \\
\text { Brasileira de Testes e } \\
\text { Dissertações (BDTD) }\end{array}$ & $\begin{array}{l}\text { "(Todos os campos:fonoforese E Todos os campos:phonophoresis E Todos os } \\
\text { campos:osteoartrite E Todos os campos:osteoarthritis E } \\
\text { Todos os } \\
\text { campos:articulação do joelho E Todos os campos:knee Joint E Todos os } \\
\text { campos:joelho E Todos os campos:knee)" }\end{array}$ \\
\hline
\end{tabular}

Fonte: Autores. 


\subsection{Fontes de informação}

O processo de busca se deu na segunda quinzena do mês de março de 2021 e foi realizada por dois pesquisadores de maneira autônoma. Os bancos de dados de estudos publicados foram a Pubmed, BVS, SciELO e PEDro. As fontes de literatura cinzenta consultadas foram a Catálogo de Teses e Dissertações da CAPES (CTD), Sumários.org e Biblioteca Digital Brasileira de Testes e Dissertações (BDTD).

\subsection{Seleção de estudos}

Foram realizadas buscas preliminares nos bancos de dados incluídos, de acordo com os critérios de inclusão determinados para a revisão, por meio da leitura do título e resumo. Os artigos que atenderam os critérios de inclusão foram reunidos e encaminhados para o Mendeley Desktop (Mendeley Ltd., Elsevier, Netherlands) e as duplicatas foram removidas. Após isso, deu-se início a uma análise rigorosa dos artigos com leitura do texto completo, que possibilitou constatar sua elegibilidade de acordo com as determinações dispostas nesta revisão.

Foi feito um registro quanto as causas que levaram a exclusão de alguns artigos disponíveis em texto completo. As discordâncias que surgiram entre os revisores durante a triagem dos artigos foram resolvidas em consonância entre os mesmos. Em casos em que não se chegou a um consenso, solicitou-se a colaboração de um terceiro revisor. Um fluxograma foi organizado de acordo para detalhar esse processo (Figura 1).

Figura 1. Fluxograma demostrando o processo de busca e seleção dos estudos.

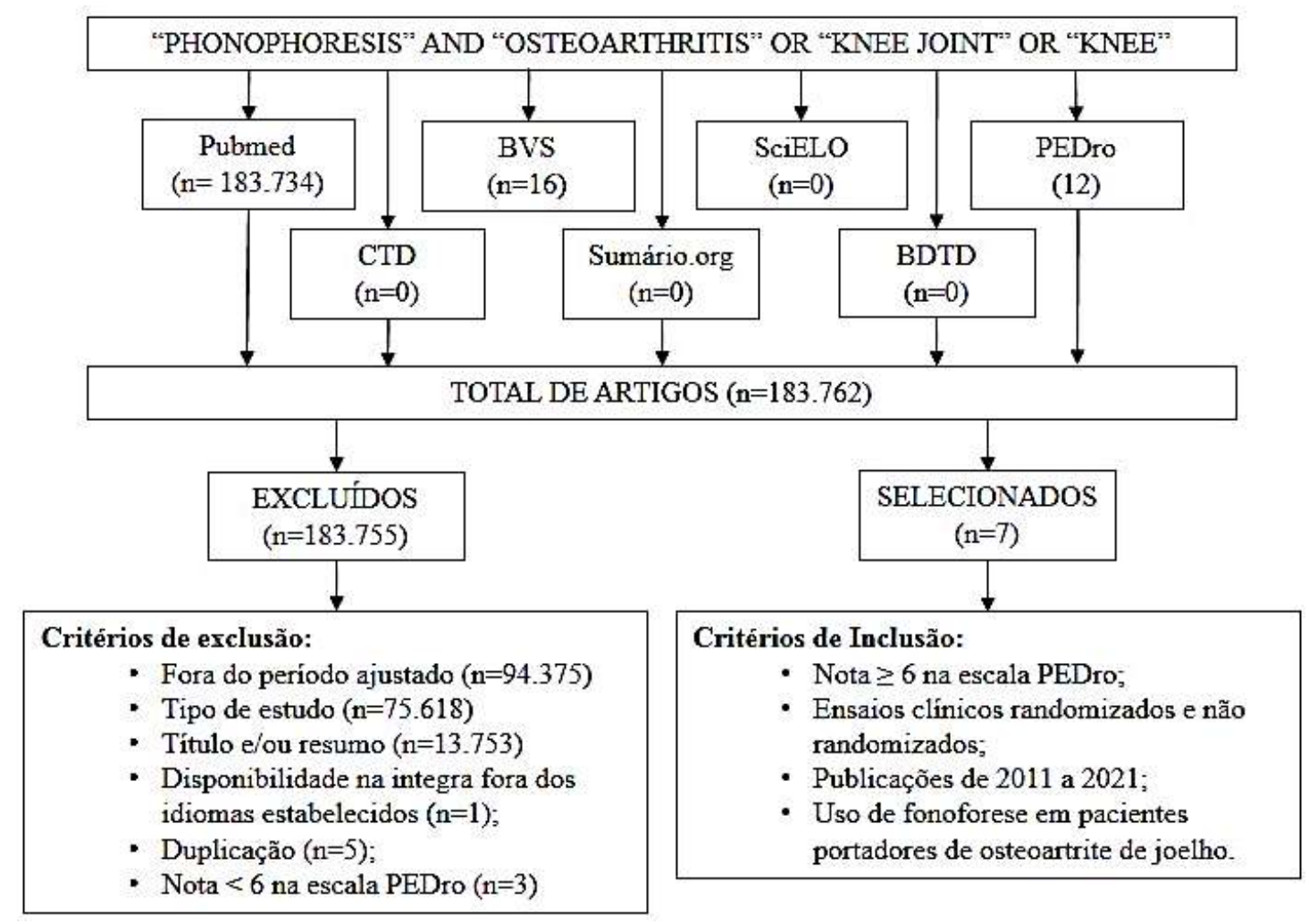

Fonte: Autores.

\subsection{Avaliação da qualidade metodológica}

A Escala PEDro em português foi utilizada para avaliação da qualidade metodológica dos artigos inclusos nesta revisão. Essa escala possui 11 itens, onde cada indicador é pontuado com um (1) ou zero ponto (0), sendo que o primeiro item não é pontuado, a pontuação se inicia somente a partir do item 2, totalizando 10 pontos (Shiwa et al., 2011). 
Os desacordos quanto a pontuação dos artigos (avaliação autônoma feita por dois colaboradores), foram resolvidas entre os revisores, e quando os mesmos não chegaram a um acordo, foi solicitada a opinião de um terceiro revisor.

Os artigos que obtiveram pontuação na escala PEDro igual ou superior a 6 foram incluídos para síntese na revisão, visando incluir apenas estudos com boa qualidade metodológica.

\subsection{Extração e apresentação dos dados}

Os dados dos estudos foram extraídos a partir de uma ferramenta criada pelos autores desta revisão, e tabulados no software Microsoft Word @ 2013 (Tabela 1). Foi considerado as seguintes informações: autores, ano de publicação, objetivo, patologia, participantes, média/faixa de idade, sexo, desenho do estudo, intervenção, caracterizas da intervenção/tratamento, principais resultados e conclusões.

Os achados desta revisão foram caracterizados em acordo com o seu objetivo, por meio de síntese descritiva e tabelas.

\section{Resultados e Discussão}

Foram obtidos a partir do emprego dos descritores um total de 183.762 estudos, sendo que após um rigoroso processo de seleção, restaram apenas 7 (0,004\%) artigos para análise e tabulação dos dados. Os estudos em sua maioria utilizaram protocolos fisioterapêuticos de fonoforese para minimizar/retarda os impactos da osteoartrite de joelho. Os principais estudos com seus respectivos parâmetros são descritos na Tabela 1.

A maioria dos protocolos descritos nos estudos utilizaram a fonoforese de forma isolada no tratamento da osteoartrite de joelho (Boyaci et al., 2013; Luksurapan et al., 2013; Zhao et al., 2016; Benlidayi et al., 2018; Boonhong et al., 2018; Pinkaew et al., 2020), sendo que somente Ahmed et al (2019), utilizou a fonoforese conjugada com estimulação elétrica nervosa transcutânea (TENS) e um programa de exercícios.

Quanto aos parâmetros da fonoforese, todos os artigos inclusos nesta revisão apresentaram configurações semelhantes em seus protocolos. Ao analisá-los, foi possível identificar os valores máximos e mínimos dos parâmetros utilizados na fonoforese: intensidade de 1 à $1,5 \mathrm{~W} / \mathrm{cm}^{2}$, frequência de 0,04 à $1 \mathrm{MHz}$, em modo contínuo, com tempo de aplicação de 5 à 30 minutos e 5 aplicações semanais, durante 2 semanas. Dentre as substâncias utilizadas estão a dexametasona, diclofenaco, ibuprofeno, piroxicam, pyllanthus amarus e um medicamento fitoterápico chinês (Boyaci et al., 2013; Luksurapan et al., 2013; Zhao et al., 2016; Benlidayi et al., 2018; Boonhong et al., 2018; Ahmed et al., 2019; Pinkaew et al., 2020).

Os estudos administraram o fármaco em forma creme ou de gel (Boyaci et al., 2013; Benlidayi et al., 2018; Boonhong et al., 2018; Ahmed et al., 2019; Pinkaew et al., 2020). De acordo com Prentice (2014), soluções em base cremosa demostram ser um mal condutor das ondas do ultrassom, entretanto, Benlidayi et al (2018), encontrou bons resultados com a utilização de ibuprofeno em creme. No mesmo estudo, a aplicação do fármaco em gel quando comparada com a aplicação em base cremosa mostrou-se estatisticamente superior na melhora dos desfechos estudados.

Todos os trabalhos evidenciaram um efeito positivo da fonoforese no alívio da dor, diminuição da rigidez articular, melhora da capacidade funcional e função física em portadores de osteoartrite de joelho, independentemente da utilização isolada ou conjugada da fonoforese com outras intervenções (Boyaci et al., 2013; Luksurapan et al., 2013; Zhao et al., 2016; Benlidayi et al., 2018; Boonhong et al., 2018; Ahmed et al., 2019; Pinkaew et al., 2020). No entanto, os estudos avaliaram esses desfechos em condições diferentes.

Luksurapan et al (2013), comparam os efeitos da fonoforese de piroxicam com o ultrassom terapêutico, evidenciaram que a fonoforese de piroxicam foi superior ao ultrassom terapêutico. Ahmed et al (2019), investigaram o efeito aditivo da fonoforese de dexametasona associada a estimulação elétrica nervosa transcutânea e um programa de exercícios de 
fortalecimento do quadríceps comparado com o ultrassom terapêutico associado a estimulação elétrica nervosa transcutânea e um programa de exercícios de fortalecimento do quadríceps, e observaram que o grupo que usou a fonoforese de dexametasona obteve uma melhora mais acentuada na dor e função do joelho. Boonhong et al (2018), compararam os efeitos do ultrassom conjugado com a estimulação elétrica nervosa transcutânea e da fonoforese de Piroxicam, e apesar de identificarem efeitos positivos nos escores de dor e funcionalidade, não identificaram diferenças entre os grupos estudados. Pinkaew et al (2020), examinaram os efeitos do tratamento com Pyllanthus amarus gel de nanopartículas aplicado por fonoforese e terapia de ultrassom, e demonstraram que a fonoforese tem efeitos mais significativos do que o ultrassom na redução da dor e melhorar a capacidade funcional. Zhao et al (2016), avaliaram os efeitos terapêuticos da fonoforese de baixa frequência com um medicamento fitoterápico chinês em comparação com o diclofenaco de sódio, e encontraram que ambos apresentam bons efeitos terapêuticos. Benlidayi et al (2018), compararam a eficácia da fonoforese do ibuprofeno em gel e creme em pacientes com osteoartrite de joelho, e observaram que fonoforese com a preparação à base de gel está associado a maior melhora clínica comparado à fonoforese em creme. Boyaci et al (2013), compararam a eficácia de três diferentes modalidades de aquecimento profundo: Fonoforese de cetoprofeno, diatermia por ondas curtas e ultrassom terapêutico, e não encontraram superioridade em nenhuma delas.

Vale ressaltar que os estudos inclusos nesta revisão apresentaram uma boa qualidade metodológica com base na escala PEDro. O trabalho com melhor qualidade metodológica foi o de Luksurapan et al (2013), com score PEDro igual a 10, seguido pelos trabalhos de Zhao et al (2016), Boonhong et al (2018), Ahmed et al (2019), Pinkaew et al (2020) com score PEDro igual a 9, Benlidayi et al (2018) e Boyaci et al (2013), com score 8 e 7, respectivamente.

Os protocolos adotados e descritos para aplicação da fonoforese evidenciaram efeitos positivos no alívio da dor e melhora da funcionalidade em indivíduos portadores de osteoartrite de joelho, tanto sendo aplicada de forma isolada quanto combinada com outras técnicas, e mesmo quando não houve diferença estatisticamente significativa entre os grupos de tratamento, a mesma mostrou trazer melhoras clinicas nas condições geradas pala osteoartrite.

Esta revisão apresenta algumas limitações como: não foi elaborado um protocolo de revisão para ser publicado e que ficasse acessível à comunidade científica, de modo a deixar maior a transparência da condução da presente revisão; haver limites de busca nos bancos de dados (fontes de informação) de 11 anos, de serem considerados somente estudos na língua inglesa, espanhola e portuguesa, bem como não terem sido realizadas buscas nas listas de referências dos estudos incluídos para síntese, podendo haver potencial viés de publicação. Também não foram utilizados métodos para minimizar erros na extração de dados como a condução dessa extração em duplicata e de forma independente. 
Tabela 1 - Resumo das vidências de uso da fonoforese em pessoas com osteoartrite de joelho.

\begin{tabular}{|c|c|c|}
\hline Autor(es)/Ano & Objetivo & Desenho/Amostra \\
\hline $\begin{array}{l}\text { Luksurapan et al. } \\
\text { (2013) }\end{array}$ & $\begin{array}{l}\text { Comparar os } \\
\text { efeitos da } \\
\text { fonoforese de } \\
\text { piroxicam com } \\
\text { ultrassom } \\
\text { terapêutico em } \\
\text { pacientes com } \\
\text { osteoartrite } \\
\text { sintomática de leve } \\
\text { a moderada } \\
\text { intensidade. }\end{array}$ & $\begin{array}{l}\text { Ensaio clínico } \\
\text { randomizado, duplo- } \\
\text { cego e controlado; } \\
\text { Amostra foi composta } \\
\text { por } 46 \text { pacientes, idade } \\
\text { média de } 58,91 \pm 10,50 \\
\text { anos. }\end{array}$ \\
\hline $\begin{array}{l}\text { Ahmed et al. } \\
(2019)\end{array}$ & $\begin{array}{l}\text { Investigar o efeito } \\
\text { aditivo da } \\
\text { fonoforese de } \\
\text { dexametasona na } \\
\text { melhora a dor e } \\
\text { função em } \\
\text { pacientes com } \\
\text { osteoartrite de } \\
\text { joelho. }\end{array}$ & $\begin{array}{l}\text { Ensaio clínico duplo- } \\
\text { cego, randomizado e } \\
\text { controlado; } \\
\text { A amostra foi } \\
\text { composta por } 46 \\
\text { mulheres, faixa etária } \\
\text { de } 40-65 \text { anos. }\end{array}$ \\
\hline
\end{tabular}

Metodologia

\section{Intervenção}

Os pacientes foram divididos em 2 grupos iguais, cada um deles composto por 23 pacientes;

G1: Recebeu fonoforese de piroxicam;

G2: Recebeu fonoforese de gel de acoplamento não-fármaco

Duração do tratamento:

Sessões: 5 por semana;

Duração: 2 semanas;

Parâmetros da Fonoforese:

Intensidade: $1 \mathrm{~W} / \mathrm{cm}^{2}$;

Frequência: $1 \mathrm{MHz}$

Modo: Contínuo;

Tempo: 10 minutos:

ERA: não especificado;

Fármaco: $20 \mathrm{mg}$ de gel de piroxicam a $0,5 \%$;

Ambos os grupos foram tratados com um programa de ultrassom usando a técnica de carícia;

A dor foi avaliada usando a escala visual analógica (EVA). O índice Western Ontario e McMaster (WOMAC) foram avaliados antes e após o tratamento em ambos os grupos.

Os pacientes foram divididos em 2 grupos iguais, cada um deles composto por 23 pacientes;

G1: Recebeu fonoforese de dexametasona, estimulação elétric nervosa transcutânea (TENS) e um programa de exercícios de fortalecimento do quadríceps;

G2: Recebeu ultrassom terapêutico, TENS e um programa de exercícios;

Duração do tratamento:

Sessões: 5 por semana;

Duração: 2 semanas;
Escore

PEDro 
Parâmetros da Fonoforese:

Intensidade: $1 \mathrm{~W} / \mathrm{cm}^{2}$;

Frequência: $1 \mathrm{MHz}$;

Modo: Contínuo;

Tempo: 10 minutos;

ERA: Não especificado;

Fármaco: Gel de dexametasona com concentração de $0,4 \%$;

Os mesmos parâmetros da TENS foram aplicados em ambos os grupos;

Parâmetros da TENS:

Modo: Não especificada;

Intensidade: Não especificada;

Frequência: $80 \mathrm{~Hz}$;

Largura de Pulso: Não especificada;

Tempo: 20 minutos;

Ambos os grupos receberam o mesmo programa de exercícios durante todas as sessões para fortalecer o músculo quadríceps. Cada terapia começou com a aplicação de compressas quentes de 20 minutos;

A dor foi avaliada usando a escala EVA e o índice WOMAC;

A mobilidade funcional foi avaliada pelo teste Timed Up and Go (TUG), WOMAC total e as subescalas de rigidez articular e função física do WOMAC.

Boonhong et al. Comparar os efeitos do
Ultrassom + TENS

à fonoforese de

Piroxicam no grau

leve a moderado de

osteoartrite

sintomática do

joelho.
Ensaio clínico duplo-

cego randomizado

controlado;

A amostra foi

composta por 61

participantes de ambos

os sexos, faixa etária de

51-80 anos.
Os pacientes foram divididos em 2 grupos, sendo 31 no grupo Ultrassom + TENS e 30 no grupo fonoforese;

G1: Recebeu ultrassom + TENS

G2: Recebeu fonoforese de Piroxicam;

Duração do tratamento:

Sessões: 5 por semana;

Duração: 2 semanas;

Parâmetros da Fonoforese:

Intensidade: $1 \mathrm{~W} / \mathrm{cm}^{2}$

Frequência: $1 \mathrm{MHz}$;

Modo: Contínuo;

Tempo: 10 minutos; intensidade da dor e mobilidade funcional no grupo fonoforese de dexametasona.
Os grupos Ultrassom + TENS Os resultados mostram que e fonoforese de Piroxicam Ultrassom + TENS e experimentaram uma melhora fonoforese de piroxicam EVA e no WOMAC total dor e na melhora da pós-tratamento $(\mathrm{P}<0,001)$. O funcionalidade,
grupo fonoforese de
diferenças significativas piroxicam teve melhores entre seus efeitos. escores de dor na EVA e no WOMAC, mas significância estatística.

(2)




$\begin{array}{ll}\text { Pinkaew et al. } & \text { Examinar os } \\ \text { (2020) } & \text { efeitos do } \\ & \text { tratamento com } \\ & \text { Pyllanthus amarus } \\ & \text { gel de } \\ & \text { nanopartículas } \\ & \text { aplicado por } \\ & \text { fonoforese e } \\ & \text { terapia de ultrasson } \\ & \text { em pacientes com } \\ & \text { osteoartrite de } \\ & \text { Joelho sintomática }\end{array}$

Ensaio clínico randomizado, duplocego e controlado;

A amostra foi composta por 30 pacientes, faixa etária 64-65 anos.

Joelho sintomática.
Zhao et al. (2016)
ERA: Não especificado;

Fármaco: Gel de piroxicam;

Os mesmos parâmetros da TENS foram aplicados em ambos os grupos;

Parâmetros da TENS:

Modo: Convencional;

Intensidade: $10 \mathrm{~mA}$ a $30 \mathrm{~mA}$;

Frequência: $100 \mathrm{~Hz}$;

Largura de Pulso: $100 \mu$ s;

Tempo: 20 minutos;

A dor foi avaliada usando a EVA e o desempenho funcional foi avaliado pelo WOMAC.

Os pacientes foram divididos em 2 grupos iguais, cada um deles composto por 15 pacientes;

G1: Recebeu fonoforese de Phyllanthus amarus;

G2: Recebeu terapia de ultrassom;

Duração do tratamento:

Sessões: 10 sessões;

Duração: Não especificado;

Parâmetros da Fonoforese:

Intensidade: $1 \mathrm{~W} / \mathrm{cm}^{2}$

\section{Frequência: $1 \mathrm{MHz}$;}

Modo: Contínuo;

Tempo: 10 minutos

ERA: Não especificado;

Fármaco: Gel de Phyllanthus amarus;

A dor foi avaliada usando a EVA e a capacidade funcional foi avaliada pelo teste de caminhada de 6 minutos (TC6M).

Os pacientes foram divididos em 3 grupos iguais, dois grupos eram compostos por 40 pacientes e um dos grupos era composto por 20 pacientes;

G1: Recebeu fonoforese de baixa frequência com medicamento fisioterápico chinês;

frequência com um

medicamento fitoterápico chinês Amostra foi composta

em comparação
Ensaio clínico duplocego, randomizado e
Os escores da EVA e o A fonoforese é sugerida TC6M mostram melhora como um método eficaz no significativa após o tratamento da osteoartrite tratamento em ambos os de joelho sintomática para grupos $(\mathrm{p}<0,05)$. Contudo o reduzir a dor e melhorar a grupo fonoforese mostrou capacidade funcional. grupo fonoforese mostrou tanto em termos de redução tanto em termos de redução
do escore da dor na EVA do escore da dor na EVA
$(\mathrm{p}<0,05)$ no TC6M $(\mathrm{p}<0,05)$ em comparação ao ultrassom terapêutico.
No grupo medicamento $\mathrm{O}$ medicamento fitoterápico fitoterápico chinês e no grupo chinês e o diclofenaco de diclofenaco de sódio foram sódio podem apresentar encontradas melhorias bons efeitos terapêuticos significativas após o para a osteoartrite de joelho tratamento em todas as em termos de alívio da dor medidas de resultado, exceto e melhoria da função física. 
com o diclofenaco ambos os sexos, faixa etária de idade de 40 osteoartrite de 75 anos.

joelho.

$\begin{array}{llll}\begin{array}{l}\text { Benlidayi } \\ \text { (2018) }\end{array} & \text { al. } & \begin{array}{l}\text { Comparar a } \\ \text { eficácia da } \\ \text { fonoforese do } \\ \text { ibuprofeno em gel } \\ \text { e creme em } \\ \text { pacientes com } \\ \text { osteoartrite de } \\ \text { joelho. }\end{array} & \begin{array}{l}\text { Ensaio clínico } \\ \text { randomizado simples } \\ \text { cego. }\end{array} \\ & \begin{array}{l}\text { A amostra foi } \\ \text { composta por } 61 \\ \text { participantes de ambos } \\ \text { os sexos, idade média } \\ \text { de } 57,9 \pm 9,7 \text { anos. }\end{array}\end{array}$

G3: Recebeu tratamento placebo;

Duração do tratamento:

rigidez e amplitude

Sessões: 5 por semana;

Duração: 2 semanas;

Parâmetros da Fonoforese:

Intensidade: Não especificado;

Frequência: 0,04 MHz;

Modo: Não especificado;

Tempo: 30 minutos;

ERA: Não especificado;

Fármaco: Grupo de diclofenaco de sódio usou-se gel de diclofenaco de sódio $10 \mathrm{mg}$; Grupo placebo usou-se $3 \mathrm{~mL}$ de cloreto de sódio; Grupo de medicamento Fitoterápico chinês usou-se $3 \mathrm{~mL}$ de uma pomada de medicamento fitoterápico;

A dor em repouso foi avaliada usando a EVA, a dor em movimento e a amplitude de movimento foram avaliadas usando o índice WOMAC.

Os pacientes foram divididos em 2 grupos, sendo 30 no grupo fonoforese em gel e 31 no grupo fonoforese em creme;

G1: Recebeu fonoforese de ibuprofeno em gel;

G2: Recebeu fonoforese ibuprofeno em creme;

Duração do tratamento:

Sessões: 5 por semana;

Duração: 2 semanas;

Parâmetros da Fonoforese:

Intensidade: $1 \mathrm{~W} / \mathrm{cm}^{2}$;

Frequência: $1 \mathrm{MHz}$;

Modo: Contínuo;

Após o tratamento, ambos os A fonoforese de ibuprofeno grupos apresentaram melhora é eficaz na redução da dor, em comparação com as rigidez e limitação medidas basais, incluindo dor funcional. Fonoforese com (EVA) e rigidez (WOMAC). a preparação à base de gel No grupo da fonoforese em de ibuprofeno está gel, a melhora no escore EVA associado a maior melhora foi maior do que a observada clínica d no grupo da fonoforese em de ibuprofeno em creme. creme (p <0,001). Da mesma forma, a melhora no escore total do WOMAC também foi
maior no grupo da fonoforese em gel $(p<0,001)$

Tempo: 5 minutos;

ERA: Não especificado;

Fármaco: Ibuprofeno com concentração de 5\%;

As medidas de desfecho principais foram avaliadas pela EVA e pelo WOMAC. 
Boyaci

(2013)

\begin{tabular}{|c|c|}
\hline $\begin{array}{l}\text { Comparar a } \\
\text { eficácia de três } \\
\text { diferentes } \\
\text { modalidades de } \\
\text { aquecimento } \\
\text { profundo: } \\
\text { Fonoforese de } \\
\text { cetoprofeno, } \\
\text { diatermia por } \\
\text { ondas curtas e } \\
\text { ultrassom } \\
\text { terapêutico na } \\
\text { osteoartrite de } \\
\text { joelho. }\end{array}$ & $\begin{array}{l}\text { A amostra foi } \\
\text { composta por } 101 \\
\text { mulheres, faixa etária } \\
\text { de } 40-65 \text { anos. }\end{array}$ \\
\hline
\end{tabular}

Os pacientes foram divididos em 3 grupos, sendo 35 no grupo diatermia por ondas curtas e 33 nos grupos fonoforese de cetoprofeno e ultrassom terapêutico;

G1: Recebeu fonoforese de cetoprofeno;

G2: Recebeu ultrassom terapêutico;

G3: Recebeu diatermia por ondas curtas;

Duração do tratamento:

Sessões: 5 por semana

Duração: 2 semanas;

Parâmetros da Fonoforese:

Intensidade: $1,5 \mathrm{~W} / \mathrm{cm}^{2}$.

Frequência: $1 \mathrm{MHz}$;

Modo: Não especificado;

Tempo: 8 minutos;

ERA: Não especificada;

Fármaco: Gel de cetoprofeno (100 mg);

Parâmetros da Diatermia por ondas Curtas:

Frequência: $27,12 \mathrm{MHz}$

Tempo: 20 minutos;

Para avaliar a eficácia do tratamento usou-se a escala visual analógica (EVA), tempo de caminhada de $15-\mathrm{m}$ e o índice Western Ontario e McMaster (WOMAC).
Os resultados do estudo A escolha de uma das mostraram que os parâmetros opções terapêuticas, seja a da EVA, tempo de caminhada fonoforese ou a diatermia de $15 \mathrm{~m}$ e índice WOMAC por ondas curtas ou melhoraram com todas as três ultrassom terapêutico, modalidades de aquecimento proporciona resultados profundo, e todas as três eficazes e nenhuma delas é modalidades foram eficazes, superior às outras. modalidades foram eficazes,
sem diferença significativa entre os três grupos em termos de eficácia

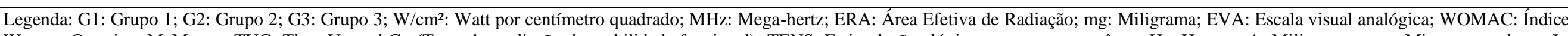

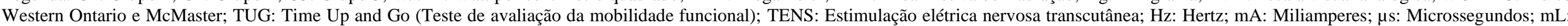
mililitros. Fonte: Autores. 


\section{Conclusão}

A fonoforese apresentou efeitos positivos para o alívio da dor e melhora da funcionalidade em indivíduos portadores de osteoartrite de joelho. Quando aos seus parâmetros, o ultrassom foi utilizado em modo contínuo, com intensidade de 1 à 1,5 $\mathrm{W} / \mathrm{cm}^{2}$, frequência de 0,04 à $1 \mathrm{MHz}$, tempo de aplicação entre 5 a 30 minutos e uma frequência de 5 aplicações semanais, durante 2 semanas, associado com os seguintes fármacos: dexametasona, diclofenaco, ibuprofeno, piroxicam, pyllanthus amarus e um medicamento fitoterápico chinês.

\section{Implicações dos resultados para a prática clínica}

Apesar de não ser próprio de uma revisão de escopo fornecer recomendações para a prática, pontuamos que a fonoforese isolada apresenta resultados eficazes no tratamento da osteoartrite de joelho, mas pode ser empregada de forma conjunta a outras modalidades terapêuticas, uma vez que, também demostra ser eficaz.

\section{Implicações dos resultados para novas pesquisas}

Recomenda-se que estudos futuros analisem se existe algum fármaco que é utilizado na iontoforese (dexametasona, diclofenaco, ibuprofeno, piroxicam), que seja mais eficaz em relação aos outros fármacos comumente utilizados nesta modalidade terapêutica, levando em conta os participantes, intervenção e desfechos abordados nesta revisão de escopo. Além disso, também preconiza-se que pesquisas futuras descrevam os parâmetros utilizados em sua totalidade, com o intuito de permitir a reprodução da técnica.

\section{Agradecimentos}

Os autores deste estudo agradecem em primeiro lugar a Deus e em segundo a Universidade Federal do Amazonas (UFAM) que proporcionaram o desenvolvimento desta revisão.

\section{Referências}

Ahmed, M. A. S., Saweeres, E. S. B., Abdelkader, N. A., Abdelmajeed, S. F., \& Fares, A. R. (2019). Improved pain and function in knee osteoarthritis with dexamethasone phonophoresis: A randomized controlled trial. Indian journal of orthopaedics, 53:700-707.

Barduzzi, G. D. O., Rocha Júnior, P. R., Souza Neto, J. C. D., \& Aveiro, M. C. (2013). Capacidade funcional de idosos com osteoartrite submetidos a fisioterapia aquática e terrestre. Fisioterapia em Movimento, 26(2): 349-360.

Benlidayi, I. C., Gokcen, N., \& Basaran, S. (2018). Comparative short-term effectiveness of ibuprofen gel and cream phonophoresis in patients with knee osteoarthritis. Rheumatology international, 38(10): 1927-1932.

Boonhong, J., Suntornpiyapan, P., \& Piriyajarukul, A. (2018). Ultrasound combined transcutaneous electrical nerve stimulation (UltraTENS) versus phonophoresis of piroxicam $(\mathrm{PhP})$ in symptomatic knee osteoarthritis: A randomized double-blind, controlled trial. Journal of back and musculoskeletal rehabilitation, 31(3), 507-513.

Boyaci, A., Tutoglu, A., Boyaci, N., Aridici, R., \& Koca, I. (2013). Comparison of the efficacy of ketoprofen phonophoresis, ultrasound, and short-wave diathermy in knee osteoarthritis. Rheumatology international, 33(11), 2811-2818.

Cavalcante, J. G. G., da Silva, R. R. C. P., Lopes, M. C. B. L., de Sousa, T. O., Sampaio, L. L., de Sousa Figueiredo, I. H., \& Lira, J. A. C. (2019). Uso da fonoforese para alívio da dor e inflamação em doenças do sistema musculoesquelético. Revista Eletrônica Acervo Saúde, (37); e1664-e1664.

Duarte, V. D. S., Santos, M. L. D., Rodrigues, K. D. A., Ramires, J. B., Arêas, G. P. T., \& Borges, G. F. (2013). Exercícios físicos e osteoartrose: uma revisão sistemática. Fisioterapia em Movimento, 26(1): 193-202.

Ito, C. B., Schneider, L. C. L., Massuda, E. M., \& Bertolini, S. M. M. G. (2019). Causas, consequências e tratamento da osteoartrite do joelho e quadril: revisão sistemática. Arquivos do MUDI, 23(3), 455-466.

Luksurapan, W., \& Boonhong, J. (2013). Effects of phonophoresis of piroxicam and ultrasound on symptomatic knee osteoarthritis. Archives of physical medicine and rehabilitation, 94(2), 250-255.

Magalhães, I. (2018). Benefícios de um programa de fortalecimento excêntrico do quadríceps no tratamento da osteoartrite de joelho. Fisioterapia Brasil, 7(1): 73-78.

Peters, M., Godfrey, C., McInerney, P., Munn, Z., Trico, A., \& Khalil, H. (2020). Chapter 11: Scoping Reviews. In E. Aromataris \& Z. Munn (Eds.), JBI 
Research, Society and Development, v. 10, n. 8, e57210817522, 2021

(CC BY 4.0) | ISSN 2525-3409 | DOI: http://dx.doi.org/10.33448/rsd-v10i8.17522

Manual for Evidence Synthesis (pp. 407-452). JBI. https://doi.org/10.46658/JBIMES-20-12.

Pinkaew, D., Kiattisin, K., Wonglangka, K., \& Awoot, P. (2020). Phonophoresis of Phyllanthus amarus nanoparticle gel improves functional capacity in individuals with knee osteoarthritis: A randomized controlled trial. Journal of bodywork and movement therapies, 24(1), 15-18.

Prentice, W. E., Underwood, F., Quillen, W. S. (2014). Modalidades terapêuticas para fisioterapeutas. (4a ed.), AMGH, 599 p.

Senna, E. R., De Barros, A. L. P., Silva, E. O., Costa, I. F., Pereira, L. V. B., Ciconelli, R. M., \& Ferraz, M. B. (2004). Prevalence of rheumatic diseases in Brazil: a study using the COPCORD approach. The Journal of rheumatology, 31(3), 594-597.

Shiwa, S. R., Costa, L. O. P., Lima Moser, A. D., Carvalho Aguiar, I., \& Oliveira, L. V. F. (2011). PEDro: a base de dados de evidências em fisioterapia. Fisioterapia em Movimento,24(3).

Silva, G. M. D., Senna, K. M. S., Sousa, E. B. D., \& Tura, B. R. (2019). Análise do impacto orçamentário da viscossuplementação no tratamento não cirúrgico da osteoartrite de joelho. Cadernos de Saúde Pública, 35, e00098618.

Vasconcelos, K. S. D. S., Dias, J. M. D., \& Dias, R. C. (2006). Relação entre intensidade de dor e capacidade funcional em indivíduos obesos com osteoartrite de joelho. Brazilian Journal of Physical Therapy, 10(2): 213-218.

Wu, Y., Zhu, S., Lv, Z., Kan, S., Wu, Q., Song, W., \& Feng, S. (2019). Effects of therapeutic ultrasound for knee osteoarthritis: a systematic review and metaanalysis. Clinical rehabilitation, 33(12), 1863-1875.

Yamada, E. F., Müller, F. A., Teixeira, L. P., \& Silva, M. D. (2018). Efeito dos exercícios de fortalecimento, de marcha e de equilíbrio no tratamento de osteoartrite de joelho. Revista Brasileira de Ciência e Movimento, 26(3): 5-13.

Zhao, J., Wang, Q., Wu, J., Shi, X., Qi, Q., Zheng, H., \& Zhang, D. (2016). Therapeutic effects of low-frequency phonophoresis with a Chinese herbal medicine versus sodium diclofenac for treatment of knee osteoarthritis: a double-blind, randomized, placebo-controlled clinical trial. Journal of traditional Chinese medicine $=$ Chung $i$ tsa chih ying wen pan, 36(5): 613-617 